\title{
Optimal Facility Location for Normally and Exponentially Distributed Points*
}

\author{
I. N. Katz** and Leon Cooper***
}

(November 12, 1975)

\begin{abstract}
$N$ destinations in the plane $\left\{P_{j}: j=1, \ldots, N\right\}$ are given as independent random variables with specified probability densities, and the problem is to find the location of the point $P$ which minimizes the expected sum of the Euclidean distances $P P_{j}$.

In this paper, upper bounds for the minimizing sum of distances are found in terms of solutions to corresponding deterministic problems and the first and second moments of the probability densities. Three commonly occurring classes of bivariate probability densities: (A) normal. (B) exponential. and (C) symmetric exponential, are then considered. Numerical tests are presented which show that in all cases, Steffensen's iteration is effective in accelerating convergence. Finally it is shown that in contrast to the deterministic case, $P$ need not be in the convex hull of the means of $P_{j}$ and a sufficient condition is given for $P$ to be in this convex hull.
\end{abstract}

Key words: Always convergent algorithms; exponentially distributed points; facility location; location theory; normally distributed points; numerical analysis; optimization; stochastic Weber problem.

\section{Introduction}

A probabilistic extension of the classical Weber (Steiner) problem was introduced in $[3]^{1}$ and [6]. $N$ destinations in the plane, $\left\{P_{j}: j=1, \ldots ., N\right\}$, are given as random variables with specified probability density functions. It is desired to find the location of a point $P$ with Cartesian coordinates $\bar{\alpha}=(\alpha, \beta)$ which minimizes the expected sum of the Euclidean distances $\overline{P P}_{j}$. A possible application of this problem to minimizing capital costs in water quality management is given in [6]. For simplicity we restrict ourselves in what follows to $E^{2}$.

Specifically, let $P_{j}$ be a random variable with probability density function $\phi_{j}\left(\bar{x}_{j}\right), \bar{x}_{j}=\left(x_{j}\right.$, $\left.y_{j}\right), j=1, \ldots, N$, where $\bar{x}_{j} \epsilon S_{j} \subseteq E^{2} . S_{j}$ is the sample space of $P_{j}$. Denote by $\left|\bar{x}-\bar{x}_{j}\right|=\left[\left(x-x_{j}\right)^{2}+\right.$ $\left.\left(y-y_{j}\right)^{2}\right]^{1 / 2}$ the Euclidean distance between $\bar{x}=(x, y)$ and $\bar{x}_{j}$. The expected value of $\overline{P P}_{j}$ is then $E\left(\left|\bar{x}-\bar{x}_{j}\right|\right)=\iint_{S_{j}}\left|\bar{x}-\bar{x}_{j}\right| \phi_{j}\left(\bar{x}_{j}\right) d x_{j} d y_{j}$ and we wish to minimize the objective function

$$
\psi(\bar{x})=\sum_{j=1}^{N} \beta_{j} \iint_{S_{j}}\left|\bar{x}-\bar{x}_{j}\right| \phi_{j}\left(\bar{x}_{j}\right) d x_{j} d y_{j}
$$

over $\bar{x}$, where the $\beta_{j}$ are given, positive weights.

In this paper $S_{j}=S$ for $j=1, \ldots, N$; that is, all $P_{j}$ are in the same sample space. We also restrict $P$ to $S$. It was shown in [6] under mild assumptions on $\phi_{j}$ that $\psi(\bar{x})$ is strictly convex, and that the minimizing point $P$ is unique. An iterative algorithm for finding $P$ was obtained formally by setting $\nabla \psi(\bar{\alpha})=0$, by "solving" this equation in the form $\bar{\alpha}=H(\bar{\alpha})$, and then by passing to the iteration scheme $\bar{x}^{n+1}=\bar{H}\left(\bar{x}^{n}\right)$ which this suggests. Specifically,

\footnotetext{
AMS Subject Classification: Primary 65K05. Secondary 90A99,90B99, 90C50.

* An invited paper. The research of the first author was supported in part by the National Science Foundation under Grant No. GJ34372.

** Present address: Department of Systems Science and Mathematics, Washington University. St. Louis, Missouri 63130. ${ }^{* * *}$ Present address: Department of Industrial Engineering and Operations Research, Southern Methodist University, Dallas, Texas
75275 .

${ }^{1}$ Figures in brackets indicate the literature references at the end of this paper.
} 


$$
\bar{x}^{n+1}=\frac{\iint_{s} \frac{\bar{\xi} G(\bar{\xi})}{\iint^{n}-\bar{\xi} \mid} d \xi d \eta}{\int \frac{G(\bar{\xi})}{\left|\bar{x}^{n}-\bar{\xi}\right|} d \xi d \eta} \equiv \bar{H}\left(\bar{x}^{n}\right) \quad n=0,1,2, \ldots
$$

where $\bar{\xi}=(\xi, \eta)$ and $G(\bar{\xi})=\sum_{j} \beta_{j} \phi_{j}(\bar{\xi})$. The algorithm was shown in [6] to be a descent method which is globally convergent to $P$ and convergence was shown to be linear, locally. Although the considerations in [6] are for $S=E^{2}$, the same proofs apply with minor variations when $S=[0, \infty)$ $\times[0, \infty)$.

In this paper, we first obtain upper bounds for

$$
\bar{\psi}=\min _{\bar{x} \epsilon S} \psi(\bar{x})
$$

in terms of the solutions to corresponding deterministic problems with the new destinations at the means of the random variables $P_{j}$. It is of interest to note that these bounds involve only the first and second moments of $\phi_{j}\left(\bar{x}_{j}\right)$. "Ne also give a sufficient condition for $P$ to be contained in an associated convex hull. This is important because whereas in the classical deterministic problem $P$ is always in the convex hull of the destinations (see [5] and [7]), a corresponding statement for the probabilistic problem is not necessarily true, as we demonstrate later with a counterexample.

Next we consider three specific classes of probability density functions:

(A) $S=E^{2}$ and $\phi_{j}\left(\bar{x}_{j}\right)=$

$\left(2 \pi \sigma_{x_{j}} \sigma_{y_{j}} \sqrt{1-\rho_{j}^{2}}\right)^{-1} \exp \left\{-\frac{1}{2 \sqrt{1-\rho_{j}^{2}}}\left[\left(\frac{x_{j}-\mu_{x_{j}}}{\sigma_{x_{j}}}\right)^{2}\right.\right.$

$$
\left.\left.-2 \rho_{j}\left(\frac{x_{j}-\mu_{x_{j}}}{\sigma_{x_{j}}}\right)\left(\frac{y_{j}-\mu_{y_{j}}}{\sigma_{y_{j}}}\right)+\left(\frac{y_{j}-\mu_{y_{j}}}{\sigma_{y_{j}}}\right)^{2}\right]\right\},
$$

the bivariate normal density function,

(B) $S=[0, \infty) \times[0, \infty)$, and

$$
\phi_{j}\left(\bar{x}_{j}\right)=\lambda_{x_{j}} \lambda_{y_{j}} \exp \left\{-\lambda_{x_{j}} x_{j}-\lambda_{y_{j}} y_{j}\right\}
$$

the bivariate exponential density function, and

(C) $S=E^{2}$ and

$$
\phi_{j}\left(\bar{x}_{j}\right)=\frac{1}{4} \lambda_{x_{j}} \lambda_{y_{j}} \exp \left\{-\lambda_{x_{j}}\left|x_{j}-\theta_{x_{j}}\right|-\lambda_{y_{j}}\left|y_{j}-\theta_{y_{j}}\right|\right\},
$$

a bivariate exponential density function with mean at $\left(\theta_{x_{j}}, \theta_{y_{j}}\right)$ and standard deviation $\left(\sqrt{2} / \lambda_{x_{j}}\right.$, $\left.\sqrt{2} / \lambda_{y_{j}}\right)$. In each case we seek the minimizing $\bar{x} \epsilon S$.

A major part of this paper deals with a special case of (A), namely the symmetric bivariate normal density when $x_{j}$ and $y_{j}$ are independent $\left(\rho_{j}=0\right)$, and $\sigma_{y_{j}}=\sigma_{y_{j}}=\sigma_{j}, j=1, \ldots ., N$. In this case, which is of considerable practical importance, all integrals appearing in (1) and (2) can be evaluated in closed form in terms of products of exponentials and modified Bessel Functions of orders 0 and 1 . This may be very useful in practical computations, since tables or subroutines can 
then be used instead of time-consuming numerical quadrature. We also prove that in this case $P$ is in the convex hull of the means of the destinations $\bar{\mu}_{j}=\left(\mu_{x_{j}}, \mu_{y_{j}}\right)$, which we denote by $\operatorname{co}\left\{\bar{\mu}_{j}\right\}$.

By means of a simple counterexample with $N=2$ we show that even if $\rho_{j}=0$, when $\sigma_{x_{j}} \neq \sigma_{y_{j}}$ $P$ need not be in co $\left\{\mu_{j}\right\}$. In the general case in (A) where the density is asymmetric, all double integrals are reducible to single integrals. This is also true for $(B)$ and $(C)$.

Finally, it was suggested in [6], that since convergence is locally linear in (2), Steffensen's iteration [4] for acceleration of convergence should be effective. We report the results of randomly generated numerical examples which confirm that this is indeed the case. We also compare our upper bound for $\bar{\psi}$ with its true value for some numerical examples.

\section{Upper Bounds for $\bar{\psi}$}

An upper bound for (3) follows from Schwarz's inequality. Since $\phi_{j}\left(\bar{x}_{j}\right) \geqslant 0$, and

$$
\iint_{S} \phi_{j}\left(\bar{x}_{j}\right) d x_{j} d y_{j}=1
$$

we have

$$
\begin{aligned}
\psi(x, y) & =\sum_{j} \beta_{j} \iint_{S} \sqrt{(x-\xi)^{2}+(y-\eta)^{2}} \phi_{j}(\xi, \eta) d \xi d \eta \\
& \leqslant \sum_{j} \beta_{j}\left[\iint_{S}\left[(x-\xi)^{2}+(y-\eta)^{2}\right] \phi_{j}(\xi, \eta) d \xi d \eta\right]_{1 / 2}\left[\iint_{S} \phi_{j}(\xi, \eta) d \xi d \eta\right]^{1 / 2} \\
& =\sum_{j} \beta_{j}\left[x^{2}-2 x \mu_{x_{j}}+E\left[x_{j}^{2}\right]+y^{2}-2 y \mu_{y_{j}}+E\left[y_{j}^{2}\right]\right]_{1 / 2} \\
& =\sum_{j} \beta_{j}\left[\left(x-\mu_{x_{j}}\right)^{2}+\left(y-\mu_{y_{j}}\right)^{2}+\sigma_{x_{j}}^{2}+\sigma_{y_{j}}^{2}\right]_{1 / 2}
\end{aligned}
$$

where $\mu_{x_{j}}=E\left[x_{j}\right], \mu_{y_{j}}=E\left[y_{j}\right]$ are the respective $x$ and $y$ means of $\phi_{j}\left(\bar{x}_{j}\right)$, and where $\sigma_{x_{j}}^{2}=E\left[x_{j}^{2}\right]-\mu_{x_{j}}^{2}, \sigma_{y_{j}}^{2}=E\left[y_{j}^{2}\right]-\bar{\mu}_{y_{j}}^{2}$ are the respective $x$ and $y$ variances of $\phi_{j}\left(\bar{x}_{j}\right)$. This gives

$$
\bar{\psi} \leqslant \min _{\bar{x} \in S} \sum_{j} \beta_{j}\left[\left(x-\mu_{x_{j}}\right)^{2}+\left(y-\mu_{y_{j}}\right)^{2}+\sigma_{j}^{2}\right]^{1 / 2}
$$

where $\sigma_{j}^{2}=\sigma_{x_{j}}^{2}+\sigma_{y_{j}}^{2}$. A coarser but more easily calculated upper bound follows immediately from (4):

$$
\begin{gathered}
\bar{\psi} \leqslant \min _{\bar{x} \in S} \sum_{j} \beta_{j}\left(\left[\left(x-\mu_{x_{j}}\right)^{2}+\left(y-\mu_{y_{j}}\right)^{2}\right]^{1 / 2}+\sigma_{j}\right) \\
=\sum_{j} \beta_{j} \sigma_{j}+\min _{\bar{x} \in S} \sum_{j} \beta_{j}\left[\left(x-\mu_{x_{j}}\right)^{2}+\left(y-\mu_{y_{j}}\right)^{2}\right]^{1 / 2}=\bar{\psi} \text { Bound. }
\end{gathered}
$$

The second term on the right in (5) is the minimum weighted sum of the distances in a deterministic problem with the destinations at the means of $P_{j}$, and the first term is a weighted sum of the standard deviations of the $P_{j}$. Note that for any $\phi_{j}\left(\bar{x}_{j}\right)$, the bounds in (4) and (5) depend only upon its first and second moments. 


\section{The Bivariate Normal Density Function}

Assume that $\phi_{j}\left(\bar{x}_{j}\right)$ for $j=1, \ldots, N$ is given by $(\mathrm{A})$. We now show that

$$
\text { if } \rho_{j}=0 \text { and } \sigma_{x_{j}}=\sigma_{y_{j}}=\sigma_{j} \text { for } j=1, \ldots, N \text { then } \bar{\alpha} \epsilon \operatorname{co}\left\{\bar{\mu}_{j}\right\}_{j=1}^{N}
$$

In this case

$$
\phi_{j}\left(\bar{x}_{j}\right)=\left(2 \pi \sigma_{j}^{2}\right)^{-1} \exp \left\{-\frac{1}{2 \sigma_{j}^{2}}\left[\left(x_{j}-\mu_{x_{j}}\right)^{2}+\left(y_{j}-\mu_{y_{j}}\right)^{2}\right]\right\} .
$$

We first prove two lemmas which are useful in locating $\bar{\alpha}$.

Lemma 1: Suppose $\mathrm{z}_{\mathrm{i}}(\overline{\mathrm{y}})$ are differentiable, real valued functions defined on $\mathrm{E}^{2}, \mathrm{i}=1, \ldots, \mathrm{N}$ and let $\bar{\gamma}$ be a point at which $\mathrm{z}(\overline{\mathrm{y}})=\Sigma \mathrm{z}_{\mathrm{i}}(\overline{\mathrm{y}})$ is minimized. Then $\bar{\gamma} \epsilon \operatorname{co}\left\{\bar{y}_{\mathrm{i}}\right\}_{\mathrm{i}=1}^{\mathrm{N}}$ is assured under the following two further assumptions:

(a) The level curves $\mathrm{S}_{i}=\left\{\overline{\mathrm{y}} \in \mathrm{E}^{2} \mid \mathrm{z}_{\mathrm{i}}(\overline{\mathrm{y}})=\mathrm{c}\right\}$ are concentric circles centered at $\overline{\mathrm{y}_{\mathrm{i}}}$, that is, $\mathrm{S}_{\mathrm{i}}=$ $\left\{\overline{\mathrm{y}}|| \overline{\mathrm{y}}-\overline{\mathrm{y}}_{\mathrm{i}} \mid=\mathrm{r}(\mathrm{c})\right\}$.

(b) For some $\mathrm{k}, \nabla \mathbf{z}_{\mathrm{k}}(\mathrm{y}) \neq 0$ if $\overline{\mathrm{y}} \neq \overline{\mathrm{y}}_{\mathrm{k}}$.

Proof: Assume, to the contrary, that $\bar{\gamma} \notin \operatorname{co}\left\{\bar{y}_{i}\right\}_{i=1}^{N}$. Then there is a line $l$ containing $\bar{\gamma}$, with a normal $\bar{a}$, such that all $\bar{y}_{i}, i=1, \ldots, N$ are contained in an open half plane bounded by $l$. For $\bar{y}$ on $l$, we have $\bar{a} \cdot \bar{y}=\bar{a} \cdot \bar{\gamma}=b$, and $\bar{a} \cdot\left(\bar{y}_{i}-\bar{\gamma}\right)>0$, for $i=1, \ldots ., N$. Now, because of (a),

$$
\nabla z_{i}(\bar{\gamma})=\left|\nabla z_{i}(\gamma)\right| \frac{\bar{\gamma}-\bar{y}_{i}}{\left|\bar{\gamma}-\bar{y}_{i}\right|}
$$

and because of (b) $\left|\nabla z_{k}(\bar{\gamma})\right|>0$. Therefore, we have

$$
(\nabla z(\bar{\gamma})) \cdot \bar{a}=\sum_{i}\left|\nabla z_{i}(\bar{\gamma})\right| \frac{\bar{\gamma}-\bar{y}_{i}}{\left|\bar{\gamma}-\bar{y}_{i}\right|} \cdot \bar{a}<0
$$

which implies that $z(\bar{y})$ is decreasing in the direction $\bar{a}$ from $\bar{\gamma}$. This contradicts the assumption that $z(\bar{y})$ is minimized at $\bar{\gamma}$, hence $\bar{\gamma} \in \operatorname{co}\left\{\bar{y}_{i}\right\}_{t=1}^{N}$.

LEMMA 2: Suppose that the probability density function $\phi_{\mathrm{j}}\left(\bar{x}_{j}\right)$ is radially symmetric about its mean $\mu_{\mathrm{j}}$; that is, suppose $\phi_{\mathrm{j}}\left(\bar{x}_{j}\right)=\mathrm{f}_{\mathrm{j}}\left(\left|\bar{x}_{j}-\bar{\mu}_{\mathrm{j}}\right|\right), \bar{x}_{\mathrm{j}} \in \mathrm{E}^{2}$. Then

$$
\mathrm{z}_{\mathrm{j}}(\bar{x})=\beta_{\mathrm{j}} \iint_{\mathrm{E}_{2}}|\bar{x}-\bar{\xi}| \phi_{\mathrm{j}}(\bar{\xi}) \mathrm{d} \xi \mathrm{d} \eta
$$

satisfies assumption (a) in lemma 1 , with $\overline{\mathbf{y}}_{\mathbf{j}}=\bar{\mu}_{\mathbf{j}}$.

Proof: Use polar coordinates $\xi-\mu_{x_{j}}=\rho \cos \theta, \eta-\mu_{y_{j}}=\rho \sin \theta$ in (8) to obtain

$$
\begin{aligned}
z_{j}(\bar{x}) & =\beta_{j} \iint_{E^{2}}|\bar{x}-\bar{\xi}| \phi_{j}(\bar{\xi}) d \xi d \eta=\beta_{j} \iint_{E_{2}}|\bar{x}-\bar{\xi}| f_{j}\left(\left|\bar{\xi}-\bar{\mu}_{j}\right|\right) d \xi d \eta \\
& =\int_{0}^{\infty} \int_{0}^{2 \pi} \rho\left[\left(x-\mu_{x_{j}}-\rho \cos \Theta\right)^{2}+\left(y-\mu_{y_{j}}-\rho \sin \Theta\right)^{2}\right]^{1 / 2} f_{j}(\rho) d \Theta d \rho \\
& =\int_{0}^{\infty} \rho f_{j}(\rho) d \rho \int_{0}^{2 \pi}\left[r_{j}^{2}+\rho^{2}-2 \rho r_{j} \cos \left(\Theta-\Phi_{j}\right)\right]^{1 / 2} d \Theta
\end{aligned}
$$

where $r_{j}^{2}=\left(x-\mu_{x_{j}}\right)^{2}+\left(y-\mu_{y_{j}}\right)^{2}$, 


$$
\cos \Phi_{j}=\left(x-\mu_{x_{j}}\right) / r_{j}, \quad \sin \Phi_{j}=\left(y-\mu_{y_{j}}\right) / r_{j}
$$

Using the periodicity of the integrand over $[0,2 \pi]$, and letting $\Psi=\theta-\Phi_{j}$, (9) becomes

$$
z_{j}(\bar{x})=\int_{0}^{\infty} \rho f_{j}(\rho) d \rho \int_{0}^{2 \pi}\left[r_{j}^{2}+\rho^{2}-2 \rho r_{j} \cos \Psi\right]^{1 / 2} d \Psi
$$

which implies that $z_{j}(\bar{x})$ in its dependence upon $\bar{x}$ is a function of $r_{j}$, The Euclidean distance to $\bar{\mu}_{j}$, alone. Hence, the level curves of $z_{j}(\bar{x})$ are concentric circles centered about $\bar{\mu}_{j}$. This proves the lemma.

Now we wish to apply lemma 1 with

$$
z_{j}(\bar{x})=\beta_{j} \iint_{E 2}|\bar{x}-\bar{\xi}| \phi_{j}(\bar{\xi}) d \xi d \eta
$$

and with $\phi_{j}(\bar{\xi})$ given by (7). It follows from [5] with $N=1$ that $z_{j}(\bar{x})$ is strictly convex on $E^{2}$. Also

$$
\nabla z_{\mathrm{j}}(\bar{x})=\beta_{j} \iint_{E 2,} \frac{\bar{x}-\bar{\xi}}{|\bar{x}-\bar{\xi}|} \phi_{j}(\bar{\xi}) d \xi d \eta,
$$

and an easy computation shows that $\nabla z_{j}\left(\bar{\mu}_{j}\right)=0$ where $\bar{\mu}_{j}=\left(\mu_{x_{j}}, \mu_{y_{j}}\right)$. Since $z_{j}(\bar{x})$ is strictly convex, $\nabla z_{j}(\bar{x}) \neq 0$ if $\bar{x} \neq \bar{\mu}_{j}$ for all $j=1, \ldots$., N. Hence $z_{j}(\bar{x})$ satisfies assumption (b) of lemma 1 . In order to show that $z_{j}(\bar{x})$ satisfies assumption (a) of lemma 1, observe that from (7) we have

$$
\phi_{j}\left(\bar{x}_{j}\right)=\left(2 \pi \sigma_{j}^{2}\right)^{-1} \exp \left\{\frac{-1}{2 \sigma_{j}^{2}}\left|\bar{x}_{j}-\bar{\mu}_{j}\right|^{2}\right\}
$$

so that $\phi_{j}\left(\bar{x}_{j}\right)=f_{j}\left(\left|\bar{x}_{j}-\bar{\mu}_{j}\right|\right)$. By lemma 2, then, $z_{j}(\bar{x})$ satisfies condition (a) of lemma 1, with $\bar{y}_{j}=\bar{\mu}_{j}$. Now apply lemma 1 with $\bar{y}_{j}=\bar{\mu}_{j}$ to obtain (6).

The result in (6) is not trivial. In the classical deterministic problem $\bar{\alpha}$ is always contained in $\operatorname{co}\left\{P_{j}\right\}_{j=1}^{N}$ where $P_{j}$ are the given destinations (see [5] and [7]). However, in the probabilistic case, we now show by means of a simple counterexample with $N=2$, that even when the $\phi_{j}$ are normal bivariate density functions with $\rho_{j}=0$, if $\sigma_{x_{j}} \neq \sigma_{y_{j}}$ then $\bar{\alpha}$ is not necessarily in $\operatorname{co}\left\{\bar{\mu}_{j}\right\}_{j=1}^{N}$.

Consider the problem: $N=2, \phi_{j}\left(\bar{x}_{j}\right)$ for $j=1,2$ given by $(\mathrm{A})$,

$$
\beta_{1}=\beta_{2}=1, \mu_{x_{1}}=1, \mu_{y_{1}}=0, \sigma_{x_{1}}=\sigma, \sigma_{y_{1}}=1 ; \mu_{x_{2}}=0, \mu_{y_{2}}=1, \sigma_{x_{2}}=1, \sigma_{y_{2}}=\sigma \text {; }
$$

$\rho_{j}=0$. In this problem $\operatorname{co}\left\{\bar{\mu}_{j}\right\}^{2}{ }_{j=1}=\{(x, y) \mid x+y=1,0 \leqslant x \leqslant 1\}$, the closed line segment joining the means. From symmetry considerations, it is easy to show that for each fixed $\sigma, 0<\sigma<\infty$, the minimizing point $\bar{\alpha}=(\alpha, \beta)$ satisfies $\alpha=\beta$. Therefore $\bar{\alpha}$ is in $\operatorname{co}\left\{\bar{\mu}_{j}\right\}_{j=1}^{2}$ if and only if $\alpha=\beta=\frac{1}{2}$. It also follows using $\nabla \psi(\bar{\alpha})=0$ that $\bar{\alpha}$ satisfies $\frac{\partial \psi}{\partial x}(\alpha, \alpha)=0$ for each $\sigma$ :

$$
\begin{gathered}
\frac{\partial \psi}{\partial x}(\alpha, \alpha)=(2 \pi \sigma)^{-1}\left\{\iint_{E^{2}} \frac{\alpha-\xi}{|\bar{\alpha}-\bar{\xi}|} \exp \left\{-\frac{1}{2}\left(\xi^{2}+\frac{(\eta-1)^{2}}{\sigma^{2}}\right)\right\} d \xi d \eta\right. \\
\left.+\iint_{E^{2}} \frac{\alpha-\xi}{\alpha-\xi} \exp \left\{-\frac{1}{2}\left(\frac{(\xi-1)^{2}}{\sigma^{2}}+\eta^{2}\right)\right\} d \xi d \eta\right\}=0 .
\end{gathered}
$$


We give the following argument: As $\sigma \rightarrow 0$ it is well known (see [2], p. 324 and [8], chapter 1, for example) that in the sense of distributions $(\sqrt{2 \pi \sigma})^{-1} \exp \left\{-1 / 2(u-1)^{2} / \sigma^{2}\right\} \rightarrow \delta(u-1)$, the Dirac-delta function centered at $u=1$. In each integral of (12), one dimension of the probabilistic problem then becomes deterministic with the corresponding coordinate of the destination fixed at 1 . Letting $\sigma \rightarrow 0$ in (12) we obtain

$$
\begin{gathered}
f(\alpha)=\int_{-\infty}^{\infty} \frac{\alpha-\xi}{\sqrt{(\alpha-\xi)^{2}+(\alpha-1)^{2}}} e^{-1 / 2 \xi^{2}} d \xi+\int_{-\infty}^{\infty} \frac{\alpha-1}{\sqrt{(\alpha-1)^{2}+(\alpha-\eta)^{2}}} e^{-1 / 2 \eta^{2}} d \eta \\
=\int_{-\infty}^{\infty} \frac{2 \alpha-\xi-1}{\sqrt{(\alpha-\xi)^{2}+(\alpha-1)^{2}}} e^{-1 / 2 \xi^{2}} d \xi=0 .
\end{gathered}
$$

We now show that the unique $\alpha$ satisfying (13), also satisfies $1 / 2<\alpha<1$. Continuity arguments then imply that for $\sigma$ sufficiently small the unique $\alpha$ satisfying (12) is also such that $1 / 2<\alpha<1$, i.e., for $\sigma$ sufficiently small $\bar{\alpha} \notin \operatorname{co}\left\{\bar{\mu}_{j}\right\}_{j=1}^{2}$, thereby providing a counterexample. We have

$$
\begin{aligned}
f\left(\frac{1}{2}\right) & =-\int_{-\infty}^{\infty} \frac{\xi}{\sqrt{\left(\xi-\frac{1}{2}\right)^{2}+\frac{1}{4}}} e^{-1 / 2 \xi^{2}} d \xi \\
& =-\int_{0}^{\infty} \xi\left(\frac{1}{\sqrt{\left(\xi-\frac{1}{2}\right)^{2}+\frac{1}{4}}}-\frac{1}{\left.\sqrt{\left(\xi+\frac{1}{2}\right)^{2}+\frac{1}{4}}\right)} e^{-1 / 2 \xi^{2}} d \xi<0\right.
\end{aligned}
$$

and

$$
f(1)=\int_{-\infty}^{\infty} \frac{1-\xi}{|1-\xi|} e^{-\frac{1}{2} \xi^{2}} d \xi=\int_{-1}^{1} e^{-\frac{1}{2} \xi^{2}} d \xi>0
$$

Hence $\alpha$ satisfying (12) is such that $\frac{1}{2}<\alpha<1$, which concludes the proof. (In fact $\alpha \rightarrow 0.66$ approximately, as $\sigma \rightarrow 0$, as seen from table 1.)

For purposes of illustration we have solved (12) numerically using an iterative scheme analogous to (2). In table 1 we give $\alpha$ for various values of $\sigma, 0<\sigma<\infty$. As $\sigma$ goes from 0 to $\infty, \alpha$ goes monotonically from 0.660 to 0 . Therefore for no value of $\sigma$ except $\sigma=1$ (when $\sigma_{x_{1}}=\sigma_{y_{1}}=1=\sigma_{x_{2}}=\sigma_{y_{2}}$ ) is $\bar{\alpha}$ in $\operatorname{co}\left\{\bar{\mu}_{j}\right\}_{j=1}^{2}$. This is in marked contrast to the deterministic case when $\bar{\alpha}$ is always in the convex hull of the destinations. We also give in table $1, \bar{\psi}$, the bound for $\bar{\psi}$ from equations (4), $\psi$ BOUND from (5), and $M$, the number of iterations of (2) to achieve three place accuracy. The bound for $\bar{\psi}$ from eq (4) was calculated from an obvious extension of the classical iteration for solving the Weber problem (see [5], or [6], for example). 
TABLE 1. Counterexample

\begin{tabular}{c|c|c|c|c|c}
\hline \hline$\sigma$ & $\alpha$ & $M$ & $\bar{\psi}$ & $\begin{array}{c}\text { Bound for } \bar{\psi} \\
\text { from (4) }\end{array}$ & $\begin{array}{c}\overline{\bar{\psi}} \text { Bound } \\
\text { from (5) }\end{array}$ \\
\hline $1 / 32$ & 0.660 & 7 & 2.123 & 2.450 & 3.415 \\
$1 / 16$ & .658 & 7 & 2.125 & 2.452 & 3.418 \\
$1 / 8$ & .650 & 7 & 2.134 & 2.462 & 3.430 \\
$1 / 4$ & .623 & 7 & 2.173 & 2.500 & 3.476 \\
$1 / 2$ & .567 & 8 & 2.326 & 2.646 & 3.650 \\
$1^{*}$ & $.500^{*}$ & - & $2.814^{*}$ & - & 4.243 \\
2 & .416 & 9 & 4.074 & 4.690 & 4.886 \\
4 & .391 & 11 & 6.969 & 8.367 & 9.660 \\
8 & .259 & 11 & 13.108 & 16.186 & 17.539 \\
16 & .122 & 12 & 25.708 & 32.094 & 33.477 \\
32 & .043 & 12 & 51.138 & 64.047 & 65.445 \\
64 & .012 & 12 & 102.136 & 128.023 & 129.430 \\
128 & .003 & 12 & 204.202 & 256.012 & 257.422 \\
256 & .001 & 12 & 408.370 & 512.006 & 513.418 \\
512 & .000 & 12 & 816.723 & 1024.003 & 1025.42 \\
\hline
\end{tabular}

*Computed from closed form expressions (see (24)).

$\bar{\psi} \mathrm{BOUND}=\sqrt{2}+2 \sqrt{1+\sigma^{2}}$.

The minimum $\psi(x, y)=\psi_{\text {min }}$ in the corresponding deterministic problem with $P_{j}$ at $\bar{\mu}_{j}$ is: $\psi_{\min }=\sqrt{2}$.

\subsection{Evaluation of Integrals in the Symmetric Uncorrelated Case}

We now give explicit closed form expressions for the evaluation of all of the integrals in (2) when $\rho_{j}=0$ and $\sigma_{x_{j}}=\sigma_{y_{j}}=\sigma_{j}$ for $j=1, \ldots, N$. In this case $\phi_{j}\left(\bar{x}_{j}\right)$ is given by (7) and the righthand side of (2) becomes

$$
\bar{H}(\bar{x})=\frac{\sum_{j} \beta_{j} \bar{N}_{j}(\bar{x})}{\sum \beta_{j} D_{j}(\bar{x})}
$$

where

$\bar{N}_{j}(\bar{x})=\left(2 \pi \sigma_{j}^{2}\right)^{-1} \int_{-\infty}^{\infty} \int_{-\infty}^{\infty} \bar{\xi}\left[(x-\xi)^{2}+(y-\eta)^{2}\right]^{-1 / 2} \exp \left\{-\frac{1}{2 \sigma_{j}}\left[\left(\xi-\mu_{x_{j}}\right)^{2}+\left(\eta-\mu_{y_{j}}\right)^{2}\right]\right\} d \xi d \eta$

$D_{j}(\bar{x})=\left(2 \pi \sigma_{j}^{2}\right)^{-1} \int_{-\infty}^{\infty} \int_{-\infty}^{\infty}\left[(x-\xi)^{2}+(y-\eta)^{2}\right]^{-1 / 2} \exp \left\{-\frac{1}{2 \sigma_{j}}\left[\left(\xi-\mu_{x_{j}}\right)^{2}+\left(\eta-\mu_{y_{j}}\right)^{2}\right]\right\} d \xi d \eta$.

We evaluate the first component of $\bar{N}_{j}(\bar{x})$, which for simplicity we denote by $N_{j}(\bar{x})$. The evaluation of the second component follows similarly. Substitute $\xi-x=\rho \cos \theta, \eta-y=\rho \sin \theta$ to obtain

$$
\begin{gathered}
N_{j}(\bar{x})=\left(2 \pi \sigma_{j}^{2}\right)^{-1} \int_{0}^{\infty} \int_{0}^{2 \pi}(x+\rho \cos \theta) \exp \left\{-\frac{1}{2 \sigma_{j}^{2}}\left[\left(x-\mu_{x_{j}}+\rho \cos \theta\right)^{2}\right.\right. \\
\left.\left.+\left(y-\mu_{y_{j}}+\rho \sin \theta\right)^{2}\right]\right\} d \theta d \rho=K_{j}(\bar{x})+L_{j}(\bar{x})
\end{gathered}
$$

Use the notation in (10) and let 


$$
\begin{aligned}
J_{j}(\rho) & =\int_{0}^{2 \pi} \exp \left\{-\left(\rho r_{j} / \sigma_{j}^{2}\right) \cos \left(\theta-\Phi_{j}\right)\right\} d \theta \\
& =\int_{0}^{2 \pi} \exp \left\{-\left(\rho r_{j} / \sigma_{j}^{2}\right) \cos \Psi\right\} d \Psi
\end{aligned}
$$

where we have substituted $\theta-\Phi_{j}=\Psi$ and used the periodicity of the integrand. Then, we have

$$
K_{j}(\bar{x})=x\left(2 \pi \sigma_{j}^{2}\right)^{-1} \exp \left\{-r_{j}^{2} / 2 \sigma_{j}^{2}\right\} \int_{0}^{\infty} \exp \left\{-\left(\rho^{2} / 2 \sigma_{j}^{2}\right)\right\} J_{j}(\rho) d \rho .
$$

Reference to [1], p. $376,9.6 .16$ or 9.6 .19 shows that

$$
J_{j}(\rho)=2 \pi I_{0}\left(\rho r_{j} / \sigma_{j}^{2}\right)
$$

where $I_{k}$ is the modified Bessel function of the first kind of order $k$. Reference to [1], p. 487, 11.4.31 then gives

$$
K_{j}(\bar{x})=\left(x / \sigma_{j}\right)(\pi / 2)^{1 / 2} \exp \left\{-r_{j}^{2} / 4 \sigma_{j}^{2}\right\} I_{0}\left\{r_{j}^{2} / 4 \sigma_{j}^{2}\right\}
$$

In order to evaluate $L_{j}(\bar{x})$ note that

$$
L_{j}(\bar{x})=\left(2 \pi \sigma_{j}^{2}\right)^{-1} \exp \left\{-r_{j}^{2} / 2 \sigma_{j}^{2}\right\} \int_{0}^{\infty} \rho \exp \left\{-\rho^{2} / 2 \sigma_{j}^{2}\right\} M_{j}(\rho) d \rho
$$

where

$$
M_{j}(\rho)=\int_{0}^{2 \pi} \cos \theta \exp \left\{\left(-\rho r_{j} / \sigma_{j}^{2}\right) \cos \left(\theta-\Phi_{j}\right)\right\} d \theta .
$$

Let $\theta-\Phi_{j}=\Psi$, use the peridoicity of the integrand and refer to [1], p. 376,9.6.19, to obtain

$$
M_{j}(\rho)=-2 \pi\left(\left(x-\mu_{x_{j}}\right) / r_{j}\right) I_{1}\left\{\rho r_{j} / \sigma_{j}^{2}\right\} .
$$

Substituting in (19), this gives

$$
L_{j}(\rho)=-\left(\left(x-\mu_{x_{j}}\right) / r_{j}\right) \exp \left\{-r_{j}^{2} / 2 \sigma_{j}^{2}\right\}\left[\sigma_{j}^{-2} \int_{0}^{\infty} \rho \exp \left\{-\rho^{2} / 2 \sigma_{j}^{2}\right\} I_{1}\left\{\rho r_{j} / \sigma_{j}^{2}\right\} d \rho\right] .
$$

Integrating by parts in the last integral, we obtain

$$
\begin{gathered}
L_{j}(\rho)=-\left(\left(x-\mu_{x_{j}}\right) / r_{j}\right) \exp \left\{-r_{j}^{2} / 2 \sigma_{j}^{2}\right\} . \\
{\left[-I_{1}\left\{\rho r_{j} / \sigma_{j}^{2}\right\} \exp \left\{-\rho^{2} /\left.2 \sigma_{j}^{2}\right|_{\rho=0} ^{\rho=\infty}+\left(r_{j} / \sigma_{j}^{2}\right) \int_{0}^{\infty} \exp \left\{-\rho^{2} / 2 \sigma_{j}^{2}\right\} I_{1}^{\prime}\left\{\rho r_{j} / \sigma_{j}^{2}\right\} d \rho\right] .\right.}
\end{gathered}
$$

The integrated term vanishes at $\rho=0$ since $I_{1}(0)=0$. At $\rho=\infty$ we use $I_{1}(z) \sim e^{z}(2 \pi z)^{-1 / 2}$ (see [1], p. 377, 9.7.1) to show that the integrated term vanishes there too. The expression in (20) can be further simplified by using $2 I_{1}^{\prime}=I_{0}+I_{2}$ (see [1], p. 376, 9.6.26). This simplification combined with use of [1], p. 487, 11.4.31 gives 


$$
L_{j}(\rho)=-\left(\left(x-\mu_{x_{j}}\right) / 2 \sigma_{j}\right)(\pi / 2)^{1 / 2} \exp \left\{-r_{j}^{2} / 4 \sigma_{j}^{2}\right\}\left(I_{0}\left\{r_{j}^{2} / 4 \sigma_{j}^{2}\right\}+I_{1}\left\{r_{j}^{2} / 4 \sigma_{j}^{2}\right\}\right)
$$

Substituting (18) and (21) in (17) gives the explicit closed form expression for a typical double integral in (14),

$$
\left.N_{j}(\bar{x})=\left(2 \sigma_{j}\right)^{-1}(\pi / 2)^{1 / 2} \exp \left\{-r_{j}^{2} / 4 \sigma_{j}^{2}\right\}\left(\left(x+\mu_{x_{j}}\right) I_{0}\left\{r_{j}^{2} / 4 \sigma_{j}^{2}\right\}-\left(x-\mu_{x_{j}}\right) I_{1}\left\{r_{j}^{2} / 4 \sigma_{j}^{2}\right)\right)\right\} .
$$

Calculations completely analogous to those which gave $K_{j}(\bar{x})$ in (18) also yield

$$
D_{j}(\bar{x})=\sigma_{j}^{-1}(\pi / 2)^{1 / 2} \exp \left\{-r_{j}^{2} / 4 \sigma_{j}^{2}\right) I_{j}\left\{r_{j}^{2} / 4 \sigma_{j}^{2}\right\} .
$$

(22) and (23) are the closed form expressions required for the iterative algorithm (2), in this special case.

The equation for the minimizing $\bar{x}, \bar{x}=\bar{H}(\bar{x})$ can now be written as

$$
\bar{x}=\frac{\frac{1}{2} \sum_{j} \beta_{j} \sigma_{j}^{-1} \exp \left\{-r_{j}^{2} / 4 \sigma_{j}^{2}\right\}\left(\left(\bar{x}+\bar{\mu}_{j}\right) I_{0}\left\{r_{j}^{2} / 4 \sigma_{j}^{2}\right\}-\left(\bar{x}-\bar{\mu}_{j}\right) I_{1}\left\{r_{j}^{2} / 4 \sigma_{j}^{2}\right\}\right)}{\sum_{j} \beta_{j} \sigma_{j}^{-1} \exp \left\{-r_{j}^{2} / 4 \sigma_{j}^{2}\right\} I_{0}\left\{r_{j}^{2} / 4 \sigma_{j}^{2}\right\}} .
$$

It is of interest to note that (24) can be rewritten in the equivalent form

$$
\bar{x}=\sum_{k=1}^{N} \lambda_{k} \bar{\mu}_{k},
$$

where

$$
\lambda_{k}=\frac{\beta_{k} \sigma_{k}^{-1} \exp \left\{-r_{k}^{2} / 4 \sigma_{k}^{2}\right\}\left(I_{0}\left\{r_{k}^{2} / 4 \sigma_{k}^{2}\right\}+I_{1}\left\{r_{k}^{2} / 4 \sigma_{k}^{2}\right\}\right)}{\sum_{j=1}^{N} \sigma_{j}^{-1} \exp \left\{-r_{j}^{2} / 4 \sigma_{j}^{2}\right\}\left(I_{0}\left\{r_{j}^{2} / 4 \sigma_{j}^{2}\right\}+I_{1}\left\{r_{j}^{2} / 4 \sigma_{j}^{2}\right\}\right)}
$$

satisfy $0<\lambda_{k}<1, \Sigma \lambda_{k}=1$. Therefore (25) is a closed form expression (in terms of $\bar{x}$ ) for $\bar{x}$ as a convex combination of $\bar{\mu}_{k}$, with constants $\lambda_{k}$. We proved earlier that in the case of bivariate normal density functions with $x_{j}$ and $y_{j}$ independent and $\sigma_{x_{j}}=\sigma_{y_{j}}=\sigma_{j}$, such a combination always exists since $\bar{x} \epsilon \operatorname{co}\left\{\bar{\mu}_{k}\right\}_{k=\mathbf{r}}^{N}$.

It is also possible to evaluate the objective function $\Psi(\bar{x})$ in (1) without numerical quadrature. We have

$$
\begin{aligned}
\psi(\bar{x}) & =\sum_{j} \beta_{j}\left(2 \pi \sigma_{j}^{2}\right)^{-1} \iint_{E^{2}}|\bar{x}-\bar{\xi}| \exp \left\{\frac{-1}{2 \sigma_{j}^{2}}\left|\bar{\xi}-\bar{\mu}_{j}\right|^{2}\right\} d \xi d \eta \\
& =\sum_{j} z_{j}(\bar{x})
\end{aligned}
$$

and

$$
\begin{aligned}
z_{j}(\bar{x}) & =\beta_{j}\left(2 \pi \sigma_{j}^{2}\right)^{-1} \exp \left\{-r_{j}^{2} / 2 \sigma_{j}^{2}\right\} \int^{\infty} \rho^{2} J_{j}(\rho) \exp \left\{-\rho^{2} / 2 \sigma_{j}^{2}\right\} d \rho \\
& =\beta_{j} \sigma_{j}^{-2} \exp \left\{-r_{j}^{2} / 2 \sigma_{j}^{2}\right\} \int_{0}^{\infty} \rho^{2} I_{0}\left(\rho r_{j} / \sigma_{j}^{2}\right) \exp \left\{-\rho^{2} / 2 \sigma_{j}^{2}\right\} d \rho .
\end{aligned}
$$


Refer to [1] p. 487, 11.4.31 and differentiate with respect to the parameter $a^{2}$ appearing there. This gives a closed form expression for the integral in (26), and (26) then becomes

$$
\begin{gathered}
z_{j}(\bar{x})=\left(\beta_{j} / \sigma_{j}\right)(\pi / 2)^{1 / 2} \exp \left\{-r_{j}^{2} / 4 \sigma_{j}^{2}\right\}\left(( r _ { j } ^ { 2 } / 2 ) \left(I_{0}\left(r_{j}^{2} / 4 \sigma_{j}^{2}\right)\right.\right. \\
\left.\left.+I_{1}\left(r_{j}^{2} / 4 \sigma_{j}^{2}\right)\right)+\sigma_{j}^{2} I_{0}\left(r_{j}^{2} / 4 \sigma_{j}^{2}\right)\right) .
\end{gathered}
$$

\subsection{Evaluation of Integrals in the General Case}

To conclude our analysis of the bivariate normal density function we now show that even in the general case (when the density is not symmetric and uncorrelated), all of the double integrals appearing in (1) and (2) can be evaluated as single integrals in terms of error functions. The iteration in (2) then becomes considerably more efficient because only single integrals need be evaluated by numerical quadrature, using subroutines available for the calculation of the error function.

In the general case $\phi_{j}\left(\bar{x}_{j}\right)$ is given by $(\mathrm{A})$ and the right-hand side of (2) becomes

$$
\bar{H}(\bar{x})=\frac{\sum_{j} \beta_{j} \bar{N}_{j}^{*}(\bar{x})}{\sum_{j} \beta_{j} D_{j}^{*}(\bar{x})}
$$

where

$$
\begin{aligned}
& \bar{N}_{j}^{*}(\bar{x})=\left(2 \pi \sigma_{x_{j}} \sigma_{y_{j}} \sqrt{1-\rho_{j}^{2}}\right)^{-1} . \\
& \int_{-\infty}^{\infty} \int_{-\infty}^{\infty} \bar{\xi}\left[(x-\xi)^{2}+(y-\eta)^{2}\right]^{-1 / 2} \exp \left\{-\frac{1}{2 \sqrt{1-\rho_{j}^{2}}}\left[\left(\frac{\xi-\mu_{x_{j}}}{\sigma_{x_{j}}}\right)^{2}\right.\right. \\
& \left.\left.-2 \rho_{j}\left(\frac{\xi-\mu_{x_{j}}}{\sigma_{x_{j}}}\right)\left(\frac{\eta-\mu_{y_{j}}}{\sigma_{y_{j}}}\right)+\left(\frac{\eta-\mu_{y_{j}}}{\sigma_{y_{j}}}\right)^{2}\right]\right\} d \xi d \eta \\
& D_{j}^{*}(\bar{x})=\left(2 \pi \sigma_{x_{j}} \sigma_{y_{j}} \sqrt{1-\rho_{j}^{2}}\right)^{-1} . \\
& \int_{-\infty}^{\infty} \int_{-\infty}^{\infty}\left[(x-\xi)^{2}+(y-\eta)^{2}\right]^{-1 / 2} \exp \left\{-\frac{1}{2 \sqrt{1-\rho_{j}^{2}}}\left[\left(\frac{\xi-\mu_{x_{j}}}{\sigma_{x_{j}}}\right)^{2}\right.\right. \\
& \left.\left.-2 \rho_{j}\left(\frac{\xi-\mu_{y_{j}}}{\sigma_{x_{j}}}\right)\left(\frac{\eta-\mu_{y_{j}}}{\sigma_{y_{j}}}\right)+\left(\frac{\eta-\mu_{y_{j}}}{\sigma_{y_{j}}}\right)^{2}\right]\right\} d \xi d \eta .
\end{aligned}
$$

Again, we evaluate only the first component of $\bar{N}_{j}^{*}(\bar{x})$ which, for simplicity, we denote by $N_{j}^{*}(\bar{x})$. The evaluation of the second component follows similarly. Substitute, as before, $\xi-x=\rho \cos \theta$, $\eta-y=\rho \sin \theta$. The exponent in brackets in (28) and (29) then becomes

$$
\begin{gathered}
{\left[\left(\frac{x-\mu_{x_{j}}+\rho \cos \theta}{\sigma_{x_{j}}}\right)^{2}-2 \rho_{j}\left(\frac{x-\mu_{x_{j}}+\rho \cos \theta}{\sigma_{x_{j}}}\right)\left(\frac{y-\mu_{y_{j}}+\rho \sin \theta}{\sigma_{y_{j}}}\right)+\left(\frac{y-\mu_{y_{j}}+\rho \sin \theta}{\sigma_{y_{j}}}\right)^{2}\right]} \\
=\rho^{2} A_{j}+2 \rho B_{j}+r_{j}^{*}=A_{j}\left(\rho+\frac{B_{j}}{A_{j}}\right)^{2}-\frac{B_{j}^{2}}{A_{j}}+r_{j}^{*}
\end{gathered}
$$


where

$$
\begin{gathered}
A_{j} \equiv A_{j}(\theta)=\frac{\cos ^{2} \theta}{\sigma_{x_{j}}^{2}}-\frac{2 \rho_{j} \cos \theta \sin \theta}{\sigma_{x_{j}} \sigma_{y_{j}}}+\frac{\sin ^{2} \theta}{\sigma_{y_{j}}^{2}} \\
B_{j} \equiv B_{j}(\theta)=\frac{\left(x-\mu_{x_{j}}\right) \cos \theta}{\sigma_{x_{j}}^{2}}-\rho_{j} \frac{\left(x-\mu_{x_{j}}\right) \sin \theta+\left(y-\mu_{y_{j}}\right) \cos \theta}{\sigma_{x_{j}} \sigma_{y_{j}}}+\frac{\left(y-\mu_{y_{j}}\right) \sin \theta}{\sigma_{y_{j}}^{2}} \\
r_{j}^{*}=\left(\frac{x-\mu_{x_{j}}}{\sigma_{x_{j}}}\right)^{2}-2 \rho_{j} \frac{\left(x-\mu_{x_{j}}\right)\left(y-\mu_{y_{j}}\right)}{\sigma_{x_{j}} \sigma_{y_{j}}}+\left(\frac{y-\mu_{y_{j}}}{\sigma_{y_{j}}}\right)^{2} \cdot
\end{gathered}
$$

Note that $A_{j} \equiv A_{j}(\theta)$ is never zero for $0<\sigma_{x_{j}}, \sigma_{y_{j}}<\infty . N_{j}^{*}(\bar{x})$ now becomes

$$
N_{j}^{*}(\bar{x})=\left(2 \pi \sigma_{x_{j}} \sigma_{y_{j}} \sqrt{1-\rho_{j}^{2}}\right)^{-1} \exp \left\{-r_{j}^{*} /\left(2 \sqrt{1-\rho_{j}^{2}}\right)\right\} \int_{0}^{2 \pi} \exp \left\{B_{j}^{2} /\left(2 A_{j} \sqrt{1-\rho_{j}^{2}}\right)\right\} P_{j}(\theta) d \theta
$$

where

$$
P_{j}(\theta)=\int_{0}^{\infty}(x+\rho \cos \theta) \exp \left\{-A_{j}\left(\rho+B_{j} / A_{j}\right)^{2} /\left(2 \sqrt{1-\rho_{j}^{2}}\right)\right\} d \rho .
$$

We now show that $P_{j}(\theta)$ can be evaluated in closed form. For each fixed $\theta$, let

$$
t=\left(\rho+\frac{B_{j}}{A_{j}}\right)\left(\frac{A_{j}}{2 \sqrt{1-\rho_{j}^{2}}}\right)^{1 / 2}
$$

then

$$
\begin{aligned}
P_{j}(\theta)= & \left(2 \sqrt{1-\rho_{j}^{2}} / A_{j}\right)^{1 / 2} . \\
& \int_{B\left(2 A, \sqrt{\left.1-\rho_{j}^{2}\right)-1 / 2}\right.}^{\infty}\left[\left(x-\frac{B_{j}}{A_{j}} \cos \theta\right)+\left(2 \sqrt{1-\rho_{j}^{2}} / A_{j}\right)^{1 / 2}(\cos \theta) t\right] e^{-t^{2}} d t \\
= & \left(\pi \sqrt{1-\rho_{j}^{2}} / 2 A_{j}\right)^{1 / 2}\left(x-\frac{B_{j}}{A_{j}} \cos \theta\right) \operatorname{erfc}\left\{B_{j} /\left(2 A_{j} \sqrt{1-\rho_{j}^{2}}\right)^{1 / 2}\right\} \\
+ & \left(\sqrt{1-\rho_{j}^{2}} / A_{j}\right)(\cos \theta) \exp \left\{-B_{j}^{2} /\left(2 A_{j} \sqrt{1-\rho_{j}^{2}}\right)\right\}
\end{aligned}
$$

where erfc is the complementary error function (defined, for instance, in [1], p. 297).

Similarly, for the terms in the denominator of (27), we have

$$
D_{j}^{*}(\bar{x})=\left(2 \pi \sigma_{x_{j}} \sigma_{y_{j}} \sqrt{1-\rho_{j}^{2}}\right)^{-1} \exp \left\{-r_{j}^{*} /\left(2 \sqrt{1-\rho_{j}^{2}}\right)\right\} \int_{0}^{2 \pi} \exp \left\{B_{j}^{2} / 2 A_{j} \sqrt{1-\rho^{2}}\right\} Q_{j}(\theta) d \theta
$$

where

$$
\begin{aligned}
Q_{j}(\theta) & =\int_{0}^{\infty} \exp \left\{-A_{j}\left(\rho+B_{j} / A_{j}\right)^{2} /\left(2 \sqrt{1-\rho_{j}^{2}}\right)\right\} d \rho \\
& =\left(\pi \sqrt{1-\rho_{j}^{2}} / 2 A_{j}\right)^{1 / 2} \operatorname{erfc}\left\{B_{j} /\left(2 A_{j} \sqrt{1-\rho_{j}^{2}}\right)^{1 / 2}\right\} .
\end{aligned}
$$


Aslo, to evaluate $\psi(\bar{x})$ in $(1)$ we have

$$
\psi(\bar{x})=\sum_{j} z_{j}(\bar{x})
$$

where

$$
\begin{array}{r}
z_{j}(\bar{x})=\beta_{j}\left(2 \pi \sigma_{x_{j}} \sigma_{y_{j}} \sqrt{1-\rho_{j}^{2}}\right)^{-1} \int_{-\infty}^{\infty} \int_{-\infty}^{\infty}\left[(x-\xi)^{2}+(y-\eta)^{2}\right]^{1 / 2}\left\{-\frac{1}{2 \sqrt{1-\rho_{j}^{2}}}\left[\left(\frac{\xi-\mu_{x_{j}}}{\sigma_{x_{j}}}\right)^{2}\right.\right. \\
\left.\left.-2 \rho_{j}\left(\frac{\xi-\mu_{x_{j}}}{\sigma_{x_{j}}}\right)\left(\frac{\eta-\mu_{y_{j}}}{\sigma_{y_{j}}}\right)+\left(\frac{\eta-\mu_{y_{j}}}{\sigma_{y_{j}}}\right)^{2}\right]\right\} d \xi d \eta .
\end{array}
$$

This leads to

$$
z_{j}(\bar{x})=\beta_{j}\left(2 \pi \sigma_{x_{j}} \sigma_{y_{j}} \sqrt{1-\rho_{j}^{2}}\right)^{-1} \exp \left\{-r_{j}^{*} /\left(2 \sqrt{1-\rho_{j}^{2}}\right)\right\} \int_{0}^{2 \pi} \exp \left\{B_{j}^{2} /\left(2 A_{j} \sqrt{1-\rho_{j}^{2}}\right)\right\} R_{j}(\theta) d \theta
$$

where

$$
R_{j}(\theta)=\int_{0}^{\infty} \rho^{2} \exp \left\{-A_{j}\left(\rho+B_{j} / A_{j}\right)^{2} /\left(2 \sqrt{1-\rho_{j}^{2}}\right)\right\} d \rho
$$

Use the substitution (30) to obtain

$$
R_{j}(\theta)=\left(2 \sqrt{1-\rho_{j}^{2}} / A_{j}\right)^{1 / 2} \int_{B_{j}\left(2 A_{j} \sqrt{\left.1-\rho_{j}^{2}\right)^{-1 / 2}}\right.}^{\infty}\left(\frac{2 \sqrt{1-\rho_{j}^{2}}}{A_{j}} t^{2}-2 \frac{B_{j}}{A_{j}}\left(\frac{2 \sqrt{1-\rho_{j}^{2}}}{A_{j}}\right)^{1 / 2} t+\frac{B_{j}^{2}}{A_{j}^{2}}\right) e^{-t^{2}} d t .
$$

Integration by parts in the first term then gives

$$
\begin{aligned}
R_{j}(\theta)=\left(2 \sqrt{1-\rho_{j}^{2}} / A_{j}\right)^{1 / 2}\left[(\sqrt{\pi / 2})\left(\frac{\sqrt{1-\rho_{j}^{2}}}{A_{j}}+\frac{B_{j}^{2}}{A_{j}^{2}}\right) \operatorname{erfc}\left\{B_{j} /\left(2 A_{j} \sqrt{1-\rho_{j}^{2}}\right)^{1 / 2}\right\}\right. \\
\left.-\frac{B_{j}}{2 A_{j}}\left(\frac{2 \sqrt{1-\rho_{j}^{2}}}{A_{j}}\right)^{1 / 2} \exp \left\{-B_{j}^{2} /\left(2 A_{j} \sqrt{1-\rho_{j}^{2}}\right)\right\}\right] .
\end{aligned}
$$

\section{The Bivariate Exponential Density Function}

In this case all double integrals appearing in (1) and (2) can be reduced to single integrals. Using the notation in (14) we have

$$
\begin{aligned}
& \bar{N}_{j}(\bar{x})=\lambda_{x_{j}} \lambda_{y_{j}} \int_{0}^{\infty} \int_{0}^{\infty} \bar{\xi}\left[(x-\xi)^{2}+(y-\eta)^{2}\right]^{-1 / 2} \exp \left\{-\left(\lambda_{x_{j}} \xi+\lambda_{y_{j}} \eta\right)\right\} d \xi d \eta \\
& D_{j}(\bar{x})=\lambda_{x_{j}} \lambda_{y_{j}} \int_{0}^{\infty} \int_{0}^{\infty}\left[(x-\xi)^{2}+(y-\eta)^{2}\right]^{-1 / 2} \exp \left\{-\left(\lambda_{x_{j}} \xi+\lambda_{y_{j}} \eta\right)\right\} d \xi d \eta .
\end{aligned}
$$

We consider only the first component of $\bar{N}_{j}(\bar{x})$ which we denote by $N_{j}(\bar{x})$. Let $\xi-x=\rho \cos \Theta$, $\eta-y=\rho \sin \Theta, \tilde{r}=x^{2}+y^{2}, \cos \tilde{\Theta}=-x / \tilde{r}, \sin \tilde{\Theta}=-y / \tilde{r}$. Thus, $\tan \tilde{\Theta}=y / x$ and $\pi \leqslant \tilde{\Theta} \leqslant 3 \pi / 2$. 
Then we obtain

$$
\begin{gathered}
N_{j}(\bar{x})=\lambda_{x_{j}} \lambda_{y_{j}}\left\{\left(\int_{0}^{\pi / 2} \int_{0}^{\infty}+\int_{\pi / 2}^{\tilde{\Theta}} \int_{0}^{-x / \cos \Theta}+\int_{\tilde{\Theta}}^{2 \pi} \int_{0}^{-y / \sin \Theta}\right)((x+\rho \cos \Theta)\right. \\
\left.\left.\exp \left\{-\lambda_{x_{j}}(x+\rho \cos \Theta)-\lambda_{y_{j}}(y+\rho \sin \Theta)\right\}\right) d \rho d \Theta\right\} \\
=\lambda_{x_{j}} \lambda_{y_{j}} \exp \left\{-x \lambda_{x_{j}}-y \lambda_{y_{j}}\right\}\left[\left(\int_{0}^{\pi / 2} \int_{0}^{\infty}+\int_{\pi / 2}^{\tilde{\Theta}} \int_{0}^{-x / \cos \Theta}+\int_{\tilde{\Theta}}^{2 \pi} \int_{0}^{-y / \sin \Theta}\right)((x+\rho \cos \Theta)\right. \\
\left.\left.\exp \left\{-\rho\left(\lambda_{x_{j}} \cos \Theta+\lambda_{y_{j}} \sin \Theta\right)\right\}\right) D \rho d \Theta\right] \\
=\lambda_{x_{j}} \lambda_{y_{j}} \exp \left\{-x \lambda_{x_{j}}-y \lambda_{y_{j}}\right\}\left[N_{1 j}(\bar{x})+N_{2 j}(\bar{x})+N_{3 j}(\bar{x})\right] .
\end{gathered}
$$

Now let

$$
\begin{aligned}
r_{j}^{2} & =\lambda_{x_{j}}^{2}+\lambda_{y_{j}}^{2} & \Psi & =\Theta-\Phi_{j} \\
\cos \Phi_{j} & =\lambda_{x_{j}} / r_{j} & \sin \Phi_{j} & =\lambda_{y_{j}} / r_{j} .
\end{aligned}
$$

Then

$$
\begin{aligned}
N_{1 j}(\bar{x}) & =\int_{0}^{\pi / 2} \int_{0}^{\infty}(x+\rho \cos \Theta) \exp \left\{-\rho r_{j} \cos \left(\Theta-\Phi_{j}\right)\right\} d \rho d \Theta \\
& =x \int_{0}^{\pi / 2} \frac{d \Theta}{r_{j} \cos \left(\Theta-\Phi_{j}\right)}+\int_{0}^{\pi / 2} \frac{\cos \Theta d \Theta}{r_{j}^{2} \cos ^{2}\left(\Theta-\Phi_{j}\right)} \\
& =\frac{x}{r_{j}} \int_{-\Phi_{j}}^{\pi / 2-\Phi_{j}} \frac{d \Psi}{\cos \Psi}+\frac{1}{r_{j}^{2}} \int_{-\Phi_{j}}^{\pi / 2-\Phi_{j}} \frac{\cos \left(\Psi+\Phi_{j}\right) d \Psi}{\cos ^{2} \Psi} \\
& =\frac{x}{r_{j}} \int_{-\Phi_{j}}^{\pi / 2-\Phi_{j}} \sec \Psi d \Psi+\frac{1}{r_{j}^{2}}\left[\cos \Phi_{j} \int_{-\Phi_{j}}^{\pi / 2 \Phi_{j}} \sec \Psi d \Psi-\sin \Phi_{j} \int_{-\Phi_{j}}^{\pi / 2 \Phi_{j}} \sec \Psi \tan \Psi d \Psi\right] \\
& =\left(\frac{x}{r_{j}}+\frac{\lambda_{x_{j}}}{r_{j}^{3}}\right) \log \left[\frac{\lambda_{x_{j}}}{\lambda_{y_{j}}}\left(\frac{r_{j}+\lambda_{x_{j}}}{r_{j}-\lambda_{y_{j}}}\right)\right]+\frac{1}{\lambda_{x_{j}} r_{j}^{2}}\left(\lambda_{y_{j}}-\lambda_{x_{j}}\right)
\end{aligned}
$$

and

$$
\begin{aligned}
N_{2 j}(\bar{x}) & =\int_{\pi / 2}^{\tilde{\Theta}} \int_{0}^{x / \cos \Theta}(x+\rho \cos \Theta) \exp \left\{-\rho\left(\lambda_{x_{j}} \cos \Theta+\lambda_{y_{j}} \sin \Theta\right)\right\} d \rho d \Theta \\
& =x \int_{\pi / 2}^{\tilde{\Theta}} \frac{1-\exp \left\{\frac{x}{\cos \Theta}\left(r_{j} \cos \left(\Theta-\Phi_{j}\right)\right\}\right.}{r_{j} \cos \left(\Theta-\Phi_{j}\right)} d \Theta \\
& +\int_{\pi / 2}^{\tilde{\Theta}} \frac{\cos \Theta\left(1-\left[\exp \left\{\frac{x}{\cos \theta} r_{j} \cos \left(\Theta-\Phi_{j}\right)\right\}\right]\left[-\frac{x}{\cos \Theta} r_{j} \cos \left(\Theta-\Phi_{j}\right)+1\right]\right) d \Theta}{r_{j}^{2} \cos ^{2}\left(\Theta-\Phi_{j}\right)}
\end{aligned}
$$




$$
\begin{aligned}
N_{3 j}(\bar{x}) & =\int_{\widetilde{\Theta}}^{2 \pi} \int_{0}^{-y / \sin \Theta}(x+\rho \cos \Theta) \exp \left\{-\rho\left(\lambda_{x_{j}} \cos \theta+\lambda_{y_{j}} \sin \theta\right)\right\} d \rho d \Theta \\
& =x \int_{\widetilde{\Theta}}^{2 \pi} \frac{1-\exp \left\{\frac{y}{\sin \theta} r_{j} \cos \left(\Theta-\Phi_{j}\right)\right\}}{r_{j} \cos \left(\Theta-\Phi_{j}\right)} d \Theta \\
& +\int_{\widetilde{\Theta}}^{2 \pi} \frac{\cos \Theta\left(1-\left[\exp \left\{\frac{y}{\sin \Theta} r_{j} \cos \left(\Theta-\Phi_{j}\right)\right\}\right]\left[-\frac{y}{\sin \Theta} r_{j} \cos \left(\Theta-\Phi_{j}\right)+1\right]\right) d \Theta}{r_{j}^{2} \cos ^{2}\left(\Theta-\Phi_{j}\right)}
\end{aligned}
$$

Entirely analogous calculations give

$$
\begin{aligned}
D_{j}(x) & =\lambda_{x_{j}} \lambda_{y_{j}}\left(\int_{0}^{\pi / 2} \int_{0}^{\infty}+\int_{\pi / 2}^{\widetilde{\Theta}} \int_{0}^{-x / \cos \Theta}+\int_{\widetilde{\Theta}}^{2 \pi} \int_{0}^{-y / \sin \Theta}\right. \\
& =\lambda_{x_{j}} \lambda_{y_{j}} \exp \left\{-x \lambda_{x_{j}}-y \lambda_{y_{j}}\right\}\left(\frac{1}{r_{j}} \log \left[\frac{\lambda_{x_{j}}}{\lambda_{y_{j}}}\left(\frac{r_{j}+\lambda_{x_{j}}}{r_{j}-\lambda_{y_{j}}}\right)\right]\right. \\
& +\int_{\pi / 2}^{\widetilde{\Theta}} \frac{1-\exp \left\{\frac{x}{\cos \Theta} r_{j} \cos \left(\Theta-\Phi_{j}\right)\right\}}{r_{j} \cos \left(\Theta-\Phi_{j}\right)} d \Theta \\
& \left.+\int_{\widetilde{\Theta}}^{2 \pi} \frac{1-\exp \left\{\frac{y}{\sin \Theta} r_{j} \cos \left(\Theta-\Phi_{j}\right)\right\}}{r_{j} \cos \left(\Theta-\Phi_{j}\right)} d \Theta\right) .
\end{aligned}
$$

In order to evaluate $\psi(\bar{x})$ use the notation in (31), where now

$$
\begin{aligned}
& z_{j}(\bar{x})=\beta_{j} \lambda_{x_{j}} \lambda_{y_{j}} \int_{0}^{\infty} \int_{0}^{\infty}\left[(x-\xi)^{2}+(y-\eta)^{2}\right]^{1 / 2} \exp \left\{-\left(\lambda_{x_{j}} \xi+\lambda_{y_{j}} \eta\right)\right\} d \xi d \eta
\end{aligned}
$$

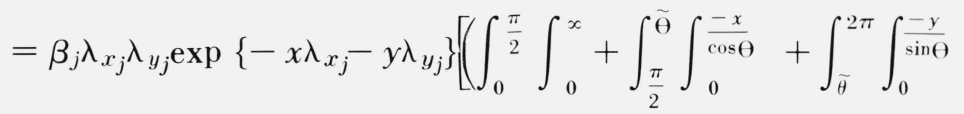

$$
\begin{aligned}
& \left.\left(\rho^{2} \exp \left\{-\rho\left(\lambda_{x_{j}} \cos \Theta+\lambda_{y_{j}} \sin \Theta\right)\right\}\right) d \rho d \Theta\right] \\
& =\beta_{j} \lambda_{x_{j}} \lambda_{y_{j}} \exp \left\{-x \lambda_{x_{j}}-y \lambda_{y_{j}}\right\}\left[z_{1 j}(\bar{x})+z_{2 j}(\bar{x})+z_{3 j}(\bar{x})\right] \text {. }
\end{aligned}
$$

Now we have

$$
\begin{gathered}
z_{1 j}(\bar{x})=\int_{0}^{\frac{\pi}{2}} \frac{2 d \Theta}{\left(\lambda_{x_{j}} \cos \Theta+\lambda_{y_{j}} \sin \Theta\right)^{3}}=2 \int_{0}^{\frac{\pi}{2}} \frac{d \Theta}{r_{j}^{3} \cos ^{3}\left(\Theta-\Phi_{j}\right)}=\frac{2}{r_{j}^{3}} \int_{-\downarrow_{j}}^{\frac{\pi}{2}-\Phi_{j}} \sec ^{3} \Psi d \Psi \\
=\frac{1}{r_{j}^{2}}\left(\frac{\lambda_{x_{j}}}{\lambda_{y_{j}^{2}}}+\frac{\lambda_{y_{j}}}{\lambda_{x_{j}^{2}}}\right)+\frac{1}{r_{j}^{3}} \log \left[\frac{\lambda_{x_{j}}}{\lambda_{y_{j}}}\left(\frac{r_{j}+\lambda_{x_{j}}}{r_{j}-\lambda_{y_{j}}}\right)\right]
\end{gathered}
$$




$$
\begin{aligned}
& z_{2 j}(\bar{x})=\int_{\frac{\pi}{2}}^{\tilde{\Theta}^{2}-\left[\exp \left\{\frac{x}{\cos \Theta} r_{j} \cos \left(\Theta-\Phi_{j}\right)\right\}\right]\left[\left(\frac{-x}{\cos \Theta^{2}} r_{j} \cos \left(\Theta-\Phi_{j}\right)+1\right)^{2}+1\right] d \Theta} \\
& r_{j}^{3} \cos ^{3}\left(\theta-\Phi_{j}\right) \\
& z_{3 j}(\bar{x})=\int_{\tilde{\Theta}}^{2 \pi} \frac{2-\left[\exp \left\{\frac{y}{\sin \Theta} r_{j} \cos \left(\Theta-\Phi_{j}\right)\right\}\right]\left[\left(\frac{-y}{\cos \Theta} r_{j} \cos \left(\Theta-\Phi_{j}\right)+1\right)^{2}+1\right] d \Theta}{r_{j}^{3} \cos ^{3}\left(\Theta^{-} \Phi_{j}\right)} .
\end{aligned}
$$

\section{The Bivariate Symmetric Exponential Density Function}

In this case all the double integrals appearing in (1) and (2) can be reduced to single integrals. Using the notation in (14) we now have

$$
\begin{aligned}
& \bar{N}_{j}(\bar{x})=(1 / 4) \lambda_{x_{j}} \lambda_{y_{j}} \int_{-\infty}^{\infty} \int_{-\infty}^{\infty} \bar{\xi}\left[(x-\xi)^{2}+(y-\eta)^{2}\right]^{-1 / 2} \exp \left\{-\lambda_{x_{j}}\left|\xi-\theta_{x_{j}}\right|-\lambda_{y_{j}}\left|\eta-\theta_{y_{j}}\right|\right\} d \xi d \eta \\
& D_{j}(\bar{x})=(1 / 4) \lambda_{x_{j}} \lambda_{y_{j}} \int_{-\infty}^{\infty} \int_{-\infty}^{\infty}\left[(x-\xi)^{2}+(y-\eta)^{2}\right]^{-1 / 2} \exp \left\{-\lambda_{x_{j}}\left|\xi-\theta_{x_{j}}\right|-\lambda_{x_{j}}\left|\eta-\theta_{y_{j}}\right|\right\} d \xi d \eta .
\end{aligned}
$$

Consider only the first component of $\bar{N}_{j}(\bar{x})$ which we again denote by $N_{j}(\bar{x})$. We have

$$
\begin{aligned}
N_{j}(\bar{x}) & =(1 / 4) \lambda_{x_{j}} \lambda_{y_{j}}\left(\int_{-\infty}^{\theta_{x_{j}}} \int_{-\infty}^{\theta_{y_{j}}} \xi|\bar{x}-\bar{\xi}|^{-1} \exp \left\{-\lambda_{x_{j}}\left(\theta_{x_{j}}-\xi\right)-\lambda_{y_{j}}\left(\theta_{y_{j}}-\eta\right)\right\} d \xi d \eta\right. \\
& +\int_{\theta_{x}}^{\infty} \int_{-\infty}^{\theta_{\nu_{j}}} \xi|\bar{x}-\bar{\xi}|^{-1} \exp \left\{-\lambda_{x_{j}}\left(\theta_{x_{j}}-\xi\right)-\lambda_{y_{j}}\left(\eta-\theta_{y_{j}}\right)\right\} d \xi d \eta \\
& +\int_{-\infty}^{\theta_{\nu_{j}}} \int_{\theta_{x_{j}}}^{\infty} \xi|\bar{x}-\bar{\xi}|^{-1} \exp \left\{-\lambda_{x_{j}}\left(\xi-\theta_{x_{j}}\right)-\lambda_{y_{j}}\left(\theta_{y_{j}}-\eta\right)\right\} d \xi d \eta \\
& \left.+\int_{\theta_{x_{j}}}^{\infty} \int_{\theta_{y_{j}}}^{\infty} \xi|\bar{x}-\bar{\xi}|^{-1} \exp \left\{-\lambda_{x_{j}}\left(\xi-\theta_{x_{j}}\right)-\lambda_{y_{j}}\left(\eta-\theta_{y_{j}}\right)\right\} d \xi d \eta\right) \\
& =(1 / 4) \lambda_{x_{j}} \lambda_{y_{j}}\left(N_{1, j}(\bar{x})+N_{2 j}(\bar{x})+N_{3 j}(\bar{x})+N_{4 j}(\bar{x})\right) .
\end{aligned}
$$

Now let $\xi-x=\rho \cos \theta, \eta-y=\rho \sin \theta, R_{j}^{2}=\left(\theta_{x_{j}}-x\right)^{2}+\left(\theta_{y_{j}}-y\right)^{2}, \cos \theta_{j}=\left(\theta_{x_{j}}-x\right) / R_{j}, \sin$ $\theta_{j}=\left(\theta_{y_{j}}-y\right) / R_{j}$. The limits of integration are determined differently depending upon whether $\left(\cos \theta_{j}, \sin \theta_{j}\right)$ is in the first, second, third, or fourth quadrant. We illustrate the procedure by calculating $N_{1 j}(\bar{x})$ assuming that $\left(\cos \theta_{j}, \sin \theta_{j}\right)$ is in the first quadrant. We then have

$$
\begin{aligned}
N_{1 j}(\bar{x})=\exp \left\{-\lambda_{x_{j}}\left(\theta_{x_{j}}-x\right)-\lambda_{y_{j}}\left(\theta_{y_{j}}-y\right)\right\} & \cdot\left\{\int_{-\frac{\pi}{2}}^{\theta_{j}} \int_{0}^{R_{j} \frac{\cos \theta_{j}}{\cos \theta}}+\int^{\pi} \int_{0}^{R} \frac{\sin \theta_{j}}{\sin \theta}+\int_{-\pi}^{-\frac{\pi}{2}} \int_{0}^{\infty}\right. \\
& \left.\left((x+\rho \cos \theta) \exp \left\{\rho\left(\lambda_{x_{j}} \cos \theta+\lambda_{y_{j}} \sin \theta\right)\right\}\right) d \rho d \theta\right\}
\end{aligned}
$$

Now let $r_{j}^{2}=\lambda_{x_{j}}^{2}+\lambda_{y_{j}}^{2}, \cos \Phi_{j}=\lambda_{x_{j}} / r_{j}, \sin \Phi_{j}=\lambda_{y_{j}} / r_{j}$ to obtain

$$
\begin{aligned}
& N_{1 j}(\bar{x})=\exp \left\{-\lambda_{x_{j}}\left(\theta_{x_{j}}-x\right)-\lambda_{y_{j}}\left(\theta_{y_{j}}-y\right)\right\} \\
& \cdot\left\{\int_{-\pi / 2}^{\theta_{j}} \int_{0}^{R_{j} \cos \theta_{j} / \cos \theta}+\int_{\theta_{j}}^{\pi} \int_{0}^{R_{j} \sin \theta_{j} / \sin \theta}+\int_{-\pi}^{-\pi / 2} \int_{0}^{\infty}\right. \\
& \quad\left((x+\rho \cos \theta) \exp \left\{\rho r_{j} \cos \left(\theta-\Phi_{j}\right)\right\}\right) d \rho d \theta .
\end{aligned}
$$


The integration with respect to $\rho$ can be performed explicitly in each term to give

$$
\begin{aligned}
& N_{1 j}(\bar{x})=\exp \left\{-\lambda_{x_{j}}\left(\theta_{x_{j}}-x\right)-\lambda_{y_{j}}\left(\theta_{y}-y\right)\right\} \\
& \cdot\left\{\int _ { - \pi / 2 } ^ { \theta _ { j } } \left[x\left(\frac{\left.\left.\exp \left\{R_{j} \frac{\cos \theta_{j}}{\cos \theta} r_{j} \cos \left(\theta-\Phi_{j}\right)\right\}\right]-1\right)}{r_{j} \cos \left(\theta-\Phi_{j}\right)}\right)\right.\right. \\
& \left.+\frac{\cos \theta}{r_{j}^{2} \cos ^{2}\left(\theta-\Phi_{j}\right)}\left(1-\left(-R_{j} \frac{\cos \theta_{j}}{\cos \theta} r_{j} \cos \left(\theta-\Phi_{j}\right)+1\right) \exp \left\{\frac{R_{j} \cos \theta_{j}}{\cos \theta} r_{j} \cos \left(\theta-\Phi_{j}\right)\right\}\right)\right] d \theta \\
& +\int_{\theta_{j}}^{\pi}\left[x\left(\frac{\left.\left.\exp \left\{R_{j} \frac{\sin \theta_{j}}{\sin \theta} r_{j} \cos \left(\theta-\Phi_{j}\right)\right\}\right]-1\right)}{r_{j} \cos \left(\theta-\Phi_{j}\right)}\right)\right. \\
& \left.+\frac{\cos \theta}{r_{j}^{2} \cos ^{2}\left(\theta-\Phi_{j}\right)}\left(1-\left(-R_{j} \frac{\sin \theta_{j}}{\sin \theta} r_{j} \cos \left(\theta-\Phi_{j}\right)+1\right) \exp \left\{R_{j} \frac{\sin \theta_{j}}{\sin \theta} r_{j} \cos \left(\theta-\Phi_{j}\right)\right\}\right)\right] d \theta \\
& +\frac{x r_{j}^{2}-\lambda_{x_{j}}}{r^{3}} \log \left(\frac{\lambda_{x_{j}}}{\lambda_{y_{j}}}\left[\frac{r_{j}+\lambda_{x_{j}}}{r_{j}-\lambda_{y_{j}}}\right]\right)-\frac{\lambda_{y_{j}}-\lambda_{x_{j}}}{\lambda_{x_{j}} r_{j}^{2}}
\end{aligned}
$$

The last two terms have been evaluated by transforming the range of integration to $\left[0, \frac{\pi}{2}\right]$ and then proceeding as in (33) and (34). Similar expressions are obtained for $N_{2 j}(\bar{x}), N_{3 j}(\bar{x}), N_{4 j}(\bar{x})$ and for the analogous terms in $D_{j}(\bar{x})$. The objective function $\psi(\bar{x})$ in (1) is evaluated analogously except that the integrand with respect to $\rho$ is of the form $\rho^{2} \exp (A \rho)$, with $A$ independent of $\rho$.

\section{Numerical Results and Discussion}

For $\phi_{j}\left(\bar{x}_{j}\right)$ given by (A), (B), and (C) we have performed a large number of numerical computations to find the minimizing $P$, and $\bar{\psi}$. In [6], we presented an example illustrating the locally linear convergence to $P$ for the case (A), bivariate normal density functions. We do the same here for the case (B), exponential density functions, and the case (C), symmetric exponential density functions. We also show how the use of Steffensen's iteration [4], accelerates the convergence. In order to emphasize the contrast between this probabilistic problem and the corresponding deterministic one, we indicate those cases in which $\bar{x} \notin$ co $\left\{\bar{\mu}_{k}\right\}_{k=1}^{N}$ with an asterisk. Comparisons are also made between $\bar{\psi}$, the bound for $\bar{\psi}$ from (4), and $\psi$ BOUND from (5).

Problem No. 1 below exhibits a typical set of data used in one of a series of problems studied numerically for case (B), the bivariate exponential density function. In this problem $N=20$, and the $\phi_{j}\left(\bar{x}_{j}\right)$ for $j=1,2, \ldots, N$ were taken as bivariate exponential density functions whose values of $\lambda_{x_{j}}$ and $\lambda_{y_{j}}$ were generated randomly. The weights $\beta_{j}$ were also generated randomly. The iteration was terminated when $\left|x_{n+1}-x_{n}\right| \leqslant 10^{-4}$ and $\left|y_{n+1}-y_{n}\right| \leqslant 10^{-4} \cdot \bar{x}_{0}$ was chosen to be

$$
\frac{\sum_{j=1}^{N} \beta_{j} \bar{\lambda}_{j}{ }^{-1}}{\sum_{j=1}^{N} \beta_{j}}
$$


where $\bar{\lambda}_{j}^{-1}=\left(\lambda_{x_{j}}^{-1}, \lambda_{y_{j}}^{-1}\right) . M$ in table 3 is the number of iterations required to achieve the desired accuracy. The computer used for all calculations was the CDC Cyber 72.

Problem No. 1

$\begin{array}{rrrrrrrrrrr}\lambda_{x_{j}}: & 1.36 & 3.56 & 13.59 & 10.33 & 6.30 & 5.37 & 15.76 & 16.43 & 4.78 & 8.94 \\ & 6.68 & 17.18 & 7.39 & 15.90 & 0.31 & 7.03 & 19.88 & 12.86 & 14.85 & 10.94 \\ & & & & & & & & & & \\ \lambda_{y_{j}}: & 7.97 & 16.21 & 8.78 & 8.82 & 15.01 & 2.50 & 0.18 & 10.35 & 18.61 & 8.07 \\ & 18.00 & 7.36 & 7.87 & 2.90 & 9.40 & 11.18 & 10.76 & 6.54 & 13.61 & 12.66 \\ & & & & & & & & & & \\ \beta_{j}: & 9.06 & 8.76 & 9.85 & 7.94 & 2.82 & 8.83 & 2.39 & 2.47 & 9.41 & 5.70 \\ & 7.36 & 9.00 & 0.94 & 0.50 & 1.27 & 0.13 & 6.49 & 5.72 & 4.28 & 9.22\end{array}$

The method of numerical solution employed is to use (2). This is discussed in more detail in [6]. Here we simply wish to illustrate the same kind of linear local convergence for the bivariate exponential density function as was shown in [6] for the bivariate normal density function. In table 2, we show this local convergence.

TABLE 2. Local convergence-bivariate exponential density function

\begin{tabular}{|c|c|c|c|c|c|c|}
\hline$n$ & $x_{n}$ & $y_{n}$ & $x_{n}-\alpha$ & $y_{n}-\beta$ & $\left|\bar{x}_{n}-\bar{\alpha}\right|$ & $\frac{\left|\bar{x}_{n+1}-\bar{\alpha}\right|}{\left|\bar{x}_{n}-\bar{\alpha}\right|}$ \\
\hline 1 & 0.326081 & 0.144027 & -0.071494 & 0.049108 & 0.08673 & 0.5442 \\
\hline 2 & .354470 & .114142 & -.043105 & .019223 & .04720 & .5983 \\
\hline 3 & .370288 & .102158 & -.027292 & .007239 & .02824 & .6317 \\
\hline 4 & .379962 & .097750 & -.017613 & .002831 & .01784 & .6373 \\
\hline 5 & .386272 & .096122 & -.011303 & .001203 & .01136 & .6356 \\
\hline 6 & .390370 & .095491 & -.007205 & .000572 & .00722 & .6191 \\
\hline 7 & .393015 & .095225 & -.004560 & .000306 & .00447 & .6398 \\
\hline 8 & .394707 & .095100 & -.002868 & .000181 & .00286 & .6049 \\
\hline 9 & .395790 & .095028 & -.001785 & .000109 & .00173 & .6300 \\
\hline 10 & .396482 & .094985 & -.001093 & .000066 & .00109 & .5963 \\
\hline 11 & .396925 & .094958 & -.000650 & .000039 & .00065 & .5692 \\
\hline 12 & .397207 & .094941 & -.000368 & .000022 & .00037 & .5135 \\
\hline 13 & .397387 & .094930 & -.000188 & .000011 & .00019 & .3684 \\
\hline 14 & .397502 & .094923 & -.000073 & .000004 & .00007 & \\
\hline
\end{tabular}

$\bar{\alpha}=(0.397575,0.094919)$.

In table 3 we indicate for 40 randomly generated problems the values of $\bar{\psi}$, the bound on $\bar{\psi}$ from (4), $\psi$ BOUND from (5), and whether or not $\bar{x}$ is in the convex hull of the means. An asterisk next to the value of $\bar{\psi}$ indicates that $\bar{x}$ is not in the convex hull of the means of distributions. The closeness of the bound is probably strongly dependent upon the nature of the data. For these randomly generated problems, the bounds are not very close.

TABLE 3. Bounds on objective function-exponential density

\begin{tabular}{c|c|c|c|c}
\hline \hline$N$ & $M$ & $\bar{\psi}$ & $\begin{array}{c}\text { Bound for } \bar{\psi} \\
\text { from (4) }\end{array}$ & $\begin{array}{c}\bar{\psi} \text { BOUND } \\
\text { from (5) }\end{array}$ \\
\hline 30 & 16 & 29.114 & 173.880 & 242.628 \\
30 & 12 & 12.079 & 100.406 & 140.618 \\
30 & 14 & $5.284^{*}$ & 55.537 & 75.538 \\
30 & 14 & 25.792 & 202.893 & 284.551 \\
30 & 6 & 12.656 & 77.019 & 106.534 \\
30 & 13 & 21.548 & 347.880 & 490.120 \\
30 & 12 & 17.742 & 118.154 & 165.042 \\
30 & 10 & 9.856 & 81.457 & 112.372 \\
30 & 10 & 7.350 & 61.793 & 83.795
\end{tabular}


TABLE 3. Bounds on objective function-exponential density-Continued

\begin{tabular}{|c|c|c|c|c|}
\hline$N$ & $M$ & $\psi$ & $\begin{array}{c}\text { Bound for } \psi \\
\text { from (4) }\end{array}$ & $\begin{array}{l}\psi \text { BOUND } \\
\text { from (5) }\end{array}$ \\
\hline 30 & 10 & 8.216 & 79.363 & 110.170 \\
\hline 20 & 14 & 5.927 & 53.949 & 74.012 \\
\hline 20 & 14 & 26.725 & 151.732 & 212.956 \\
\hline 20 & 14 & 8.522 & 68.464 & 95.960 \\
\hline 20 & 11 & 3.125 & 39.150 & 53.083 \\
\hline 20 & 20 & 23.835 & 168.143 & 236.237 \\
\hline 20 & 10 & 4.149 & 51.055 & 70.417 \\
\hline 20 & 8 & $9.196^{*}$ & 54.956 & 75.920 \\
\hline 20 & 14 & 22.643 & 344.637 & 486.403 \\
\hline 20 & 9 & 2.400 & 25.562 & 34.767 \\
\hline 20 & 6 & 16.436 & 105.702 & 147.579 \\
\hline 10 & 10 & $1.121^{*}$ & 12.074 & 15.948 \\
\hline 10 & 11 & $3.318^{*}$ & 21.399 & 29.313 \\
\hline 10 & 9 & $0.989 *$ & 12.832 & 16.929 \\
\hline 10 & 10 & $5.497^{*}$ & 47.132 & 65.906 \\
\hline 10 & 9 & 1.624 & 20.033 & 27.293 \\
\hline 10 & 14 & $1.382^{*}$ & 13.027 & 17.314 \\
\hline 10 & 10 & 4.314 & 28.651 & 39.116 \\
\hline 10 & 14 & 5.008 & 43.205 & 59.725 \\
\hline 10 & 15 & $1.140^{*}$ & 10.676 & 14.489 \\
\hline 10 & 13 & 1.773 & 24.635 & 34.190 \\
\hline 5 & 15 & $2.271^{*}$ & 14.688 & 20.107 \\
\hline 5 & 9 & $1.670^{*}$ & 26.527 & 36.952 \\
\hline 5 & 7 & $0.240^{*}$ & 3.334 & 4.015 \\
\hline 5 & 13 & 21.036 & 106.369 & 150.094 \\
\hline 5 & 18 & 2.140 & 13.529 & 18.449 \\
\hline 5 & 8 & $1.075 *$ & 18.091 & 25.193 \\
\hline 5 & 14 & $2.423^{*}$ & 13.535 & 18.639 \\
\hline 5 & 28 & 4.310 & 26.844 & 37.449 \\
\hline 5 & 17 & $1.158^{*}$ & 12.988 & 16.715 \\
\hline 5 & 15 & $1.438^{*}$ & 8.857 & 12.148 \\
\hline
\end{tabular}

Problem No. 2 below exhibits a typical set of data for the problems of case (C), the bivariate symmetric exponential function. In this problem $N=10$, and the $\phi_{j}\left(\bar{x}_{j}\right)$ for $j=1,2, \ldots ., N$ were taken as bivariate symmetric exponential density functions whose values of $\lambda_{x_{j}}, \lambda_{y_{j}}, \theta_{x_{j}}, \theta_{y_{j}}$ were generated randomly. The weights $\beta_{j}$ were similarly generated. The iteration was terminated when $\left|x_{n+1}-x_{n}\right| \leqslant 10^{-4}$ and $\left|y_{n+1}-y_{n}\right| \leqslant 10^{-4} . \bar{x}_{0}$ was chosen to be:

$$
\frac{\sum_{j=1}^{N} \beta_{j} \bar{\theta}_{j}}{\sum_{j=1}^{N} \beta_{j}}
$$

where $\bar{\theta}_{j}=\left(\theta_{x_{j}}, \theta_{y_{j}}\right) . M$ in table 5 is the number of iterations required to achieve the desired accuracy.

Problem No. 2

$$
\begin{array}{lllllllllll}
\lambda_{x_{j}}: & 1.36 & 17.53 & 8.82 & 5.37 & 4.78 & 18.61 & 6.68 & 18.00 & 2.90 & 7.03 \\
\lambda_{y_{j}}: & 7.97 & 13.59 & 15.89 & 2.50 & 16.43 & 18.82 & 18.00 & 7.39 & 1.01 & 11.18 \\
\theta_{x_{j}}: & 9.06 & 4.39 & 3.15 & 8.83 & 5.18 & 4.47 & 7.36 & 3.94 & 0.15 & 0.13 \\
\theta_{y_{j}}: & 1.78 & 9.85 & 7.51 & 7.88 & 2.47 & 4.04 & 8.59 & 0.94 & 4.70 & 9.94 \\
\beta_{j}: & 8.11 & 5.17 & 2.82 & 0.09 & 2.39 & 5.70 & 3.68 & 7.95 & 1.27 & 5.38
\end{array}
$$


Local convergence for Problem No. 2 is exhibited in table 4. In table 5 we indicate the bounds and whether or not the solution is in the convex hull of the means in the same fashion as we did for the bivariate symmetric exponential density function. It is similar to table 3 . For the problems generated for the symmetric exponential case we never found one in the convex hull of the means.

TABLE 4. Local convergence-bivariate symmetric exponential density function

\begin{tabular}{c|c|c|c|c|c|c}
\hline \hline$n$ & $x_{n}$ & $y_{n}$ & $x_{n}-\alpha$ & $y_{n}-\beta$ & $\left|\bar{x}_{n}-\bar{\alpha}\right|$ & $\frac{\left|\bar{x}_{n+1}-\bar{\alpha}\right|}{\left|\bar{x}_{n}-\bar{\alpha}\right|}$ \\
\hline 1 & 0.01668185 & 0.04066205 & 0.00462450 & 0.03773900 & 0.0380213 & 0.3519 \\
2 & .01158224 & .01629392 & -0.00047511 & .01337087 & .0133793 & .3051 \\
3 & .01210797 & .00700439 & .00005062 & .00408134 & .00408165 & .3864 \\
4 & .01255962 & .00441807 & .00050227 & .00149502 & .00157714 & .5018 \\
5 & .01253156 & .00355670 & .00047421 & .00063365 & .000791446 & .5563 \\
6 & .01238560 & .00321650 & .00032825 & .00029345 & .000440296 & .5251 \\
7 & .01224484 & .00305836 & .00018749 & .00013531 & .000231217 & .4034 \\
8 & .01213578 & .00297353 & .00007843 & .00005048 & .0000932711 &. \\
\hline
\end{tabular}

$\bar{\alpha}=(0.01205735,0.00292305)$.

TABLE 5. Bounds on objective function-symmetric exponential density

\begin{tabular}{|c|c|c|c|c|}
\hline$N$ & $M$ & $\bar{\psi}$ & $\begin{array}{l}\text { Bound for } \bar{\psi} \\
\quad \text { from (4) }\end{array}$ & $\begin{array}{l}\bar{\psi} \text { Bound } \\
\text { from (5) }\end{array}$ \\
\hline 30 & 13 & $38.195^{*}$ & 485.942 & 533.053 \\
\hline 30 & 19 & $36.889^{*}$ & 609.044 & 699.347 \\
\hline 30 & 11 & $31.590^{*}$ & 623.526 & 696.993 \\
\hline 30 & 14 & $15.391 *$ & 513.657 & 569.532 \\
\hline 30 & 13 & $26.269^{*}$ & 850.293 & 978.537 \\
\hline 30 & 11 & $3.362^{*}$ & 527.453 & 596.923 \\
\hline 30 & 13 & $15.910^{*}$ & 754.403 & 823.259 \\
\hline 30 & 28 & $54.418^{*}$ & 553.564 & 611.917 \\
\hline 30 & 10 & $56.257^{*}$ & 723.158 & 801.155 \\
\hline 30 & 12 & $7.493^{*}$ & 482.261 & 544.145 \\
\hline 20 & 16 & $0.02035^{*}$ & 324.837 & 349.834 \\
\hline 20 & 17 & $119.638^{*}$ & 503.372 & 565.895 \\
\hline 20 & 10 & $5.548^{*}$ & 312.673 & 350.797 \\
\hline 20 & 12 & $10.965^{*}$ & 369.864 & 420.667 \\
\hline 20 & 16 & $17.922^{*}$ & 469.078 & 527.044 \\
\hline 20 & 25 & $199.676^{*}$ & 682.171 & 762.248 \\
\hline 20 & 13 & $72.808^{*}$ & 553.628 & 631.301 \\
\hline 20 & 21 & $2.169^{*}$ & 215.444 & 241.296 \\
\hline 20 & 9 & $4.340^{*}$ & 452.851 & 483.389 \\
\hline 20 & 13 & $0.45620^{*}$ & 342.029 & 376.407 \\
\hline 10 & 8 & $2.407^{*}$ & 178.300 & 193.276 \\
\hline 10 & 17 & $29.185^{*}$ & 308.299 & 366.341 \\
\hline 10 & 15 & $4.695^{*}$ & 107.535 & 117.199 \\
\hline 10 & 9 & $0.47852 *$ & 177.145 & 187.936 \\
\hline 10 & 12 & $5.742^{*}$ & 237.441 & 254.580 \\
\hline 10 & 19 & $25.345^{*}$ & 205.296 & 240.394 \\
\hline 10 & 12 & $9.546^{*}$ & 186.705 & 203.622 \\
\hline 10 & 12 & $1.429^{*}$ & 140.736 & 150.786 \\
\hline 10 & 20 & $2.901 *$ & 155.144 & 178.605 \\
\hline 10 & 5 & $0.77443^{*}$ & 286.310 & 307.411 \\
\hline 5 & 12 & $9.236^{*}$ & 93.932 & 124.836 \\
\hline 5 & 25 & $0.00133^{*}$ & 49.655 & 55.333 \\
\hline 5 & 4 & $1.951^{*}$ & 85.311 & 94.246 \\
\hline 5 & 19 & $14.127^{*}$ & 216.730 & 273.959 \\
\hline 5 & 8 & $0.00000^{*}$ & 88.316 & 93.744 \\
\hline 5 & 8 & $2.465^{*}$ & 60.637 & 67.276 \\
\hline 5 & 17 & $3.493^{*}$ & 100.387 & 110.473 \\
\hline 5 & 13 & $1.301^{*}$ & 80.547 & 85.485 \\
\hline 5 & 23 & $30.839 *$ & 69.109 & 79.990 \\
\hline 5 & 13 & $0.00000^{*}$ & 31.311 & 35.560 \\
\hline
\end{tabular}




\subsection{Steffensen's Iteration}

A well known method for accelerating the convergence of linearly convergent iterative schemes is Steffensen's iteration [4]. In the case when the unknown vector is two dimensional, Steffensen's iteration, applied to the scheme in (2), is described as follows (see [4], p. 115 for a derivation):

Choose a vector $\bar{x}^{(0)}=\left(x^{(0)}, y^{(0)}\right)$, and construct the sequence of vectors $\left\{\bar{x}^{(k)}\right\}$ by the rule: for each $k=0,1,2, \ldots$, set $\bar{x}_{0}=\bar{x}^{(k)}$, and calculate $\bar{x}_{1}, \bar{x}_{2}, \bar{x}_{3}$ from

$$
\bar{x}_{n+1}=\bar{H}\left(x_{n}\right) \quad n=0,1,2 .
$$

Let the matrix $X_{n}$ be defined by

$$
X_{n}=\left(\begin{array}{ll}
x_{n} & x_{n+1} \\
y_{n} & y_{n+1}
\end{array}\right)
$$

and let $\Delta$ denote the forward difference operator. The next iterate is given by

$$
\bar{x}^{(k+1)}=\bar{x}_{0}-\Delta X_{0}\left(\Delta^{2} X_{0}\right)^{-1} \Delta \bar{x}_{0} .
$$

In order to test the utility of Steffensen's iteration in reducing the amount of calculation to converge to any given degree of tolerance, ten, 20 destination problems and ten, 10 destination problems were run on the CDC Cyber 72 both with and without the use of Steffensen's iteration

TABLE 6. Effect of Steffensen's iteration on convergence

\begin{tabular}{c|c|c}
\hline \hline $\begin{array}{c}\text { Number of } \\
\text { destinations }\end{array}$ & $\begin{array}{c}\text { Number of } \\
\text { iterations }\end{array}$ & $\begin{array}{c}\text { Number of iterations } \\
\text { (Steffensen) }\end{array}$ \\
\hline 20 & 14 & 7 \\
20 & 14 & 13 \\
20 & 16 & 6 \\
20 & 16 & 13 \\
20 & 17 & 15 \\
20 & 14 & 6 \\
20 & 12 & 5 \\
20 & 15 & 5 \\
20 & 15 & 9 \\
20 & 13 & 6 \\
20 & 14 & 6 \\
10 & 15 & 12 \\
10 & 15 & 7 \\
10 & 16 & 6 \\
10 & 16 & 8 \\
10 & 18 & 9 \\
10 & 12 & 10 \\
10 & 17 & 10 \\
10 & 13 & 8 \\
10 & 25 & 13 \\
10 & 16 & 7 \\
\end{tabular}

technique. The number of iterations given in table 6 is the total number of iterations, i.e., the number reported using Steffensen's iteration includes the three iterations required to use the method. The problems all used a bivariate normal density function. The average ratio of the number of iterations with the Steffensen method to the number of iterations without the method for the 20 problems of table 6 was 0.571 . Hence, it can be readily seen that it is quite advantageous to use the Steffensen iteration method when performing these calculations.

We thank the reviewer for his helpful suggestions and for his careful verification of many of the formulas presented in this paper. 


\section{References}

[1] Abramowitz, M., and Stegun, I., Handbook of Mathematical Functions, Nat. Bur. Stand. (U.S.), Appl. Math. Ser. 55.

[2] Arfken, G., Mathematical Methods for Physicists (Academic Press, New York, N.Y., 1968).

[3] Cooper, L., A random locational equilibrium problem, Journal of Regional Science, Vol. 14, No. 1, 47-54. (1974).

[4] Henrici, P., Elements of Numerical Analysis (John Wiley and Sons, New York, N.Y., 1966).

[5] Katz, I. N., On the convergence of a numerical scheme for solving some locational equilibrium problems, SIAM J. Appl. Math, 17, 1224-1231 (1969).

[6] Katz, I. N., and Cooper, L., An always-convergent numerical scheme for a random locational equilibrium problem, SIAM J. Num. Anal. Vol. 11, No. 4, 683-692 (Sept. 1974).

[7] Kuhn, H. W., A note on Fermat's problem, Math. Programming, No. 4, 98-107 (1973).

[8] Stakgold, I., Boundary Value Problems of Mathematical Physics, Vol. I (The Macmillan Co., New York, N.Y., 1967).

(Paper 80B1-432) 